\title{
Application of Deep Learning For Sentiment Analysis
}

\author{
Neha Sharma \\ Research Scholar \\ Department of CSE \\ Rabindranath Tagore University - \\ Bhopal, Madhya Pradesh, India \\ sharmaneha94@gmail.com
}

\author{
Dr. S Veenadhari \\ Professor \\ Department of CSE \\ Rabindranath Tagore University - \\ Bhopal, Madhya Pradesh, India
}

\author{
Rachna Kulhare \\ Research Scholar \\ Department of CSE \\ Rabindranath Tagore University - \\ Bhopal, Madhya Pradesh, India
}

\begin{abstract}
Deep learning is a type of artificial intelligence that employs neural networks, a multi-layered structure of algorithms. Deep learning is an accumulation of artificial intelligence statistics based on artificial neural networks for the teaching of functional hierarchies. In sentiment analysis, deep learning is also applied. This paper begins with an overview of deep learning before moving on to a detailed examination of its present uses in sentiment analysis.

Keywords-AI, Neural Network, Sentiment Analysis, Deep Learning.
\end{abstract}

\section{INTRODUCTION}

The process of detecting positive or negative sentiment in text is known as sentiment analysis. Clients are expressing their ideas and sentiments more freely than ever before, and emotion recognition is quickly to become an indispensable tool for tracking and understanding that opinion. Brands can learn what makes customers happy or unhappy by automatically analyzing customer feedback, such as opinions in survey responses and social media conversations. This enables them to tailor goods and services to satisfy their users' requirements.

\section{TyPES OF SENTIMENT ANALYSIS}

Models of sentiment analysis concentrate on polarity (neutral, positive, and negative), as well as emotions and feelings (sad, angry, glad, etc.), urgency (not urgent, urgent), and even intents (not interested, interested).

1. Precision in polarity is critical in business. The following are the polarity categories: Sentiment analysis, which is very pleasant, helps to determine feelings such as joy, annoyance, rage, and melancholy.

2. "The battery life of this camera is very short," for example, an aspect-based classifier would be possible to ascertain that the statement communicates a negative judgment regarding the attribute standby time.

3. Preprocessing and resources are required for multilingual sentiment analysis. The majority of such tools are available on the internet; others must be generated, and you must be able to compute in order to use it.

\section{BENEFITS OF SENTIMENT ANALYSIS INCLUDE}

1. Sorting data from tens of thousands of tweets, customer support conversations, or surveys? There is simply too much corporate data to manually process. Sentiment analysis aids firms in efficiently and cost-effectively processing large amounts of data.

2. Authentic Method can identify key concerns in real-time, such as whether a media platforms PR disaster is developing. Is a disgruntled consumer about to leave? Sentiment analysis methods can assist you in quickly identifying these types of circumstances so that you can take appropriate action.

\section{DEEP LEARNING}

Deep learning is a type of artificial intelligence that is intentionally programmed. In order to execute feature extraction and transformation, it employs a number of nonlinear processing units. Each of the subsequent layers uses the output from the previous layer as input.

\section{Limitations}

1. It only learns through the observations.

2. It comprises of biases issues.

\section{Advantages}

1. It lessens the need for feature engineering

2. It eradicates all those costs that are needless.

3. It easily identifies difficult defects.

4. It results in the best-in-class performance on problems.

\section{Disadvantages}

1. It requires an ample amount of data.

2. It is quite expensive to train.

3. It does not have strong theoretical groundwork.

\section{DeEP LEARNING APPLICATION IN SENTIMENT ANALYSIS}

\section{A. Sentiment Analysis Based of Customer Reviews}

Deep Learning based Sentiment Analysis (DL-SA) is implemented to achieve framework for sentiment analysis. 
Sentiment analysis results in identifying reviews as positive or negative. the solution is based on supervised learning method which needs training data [1].

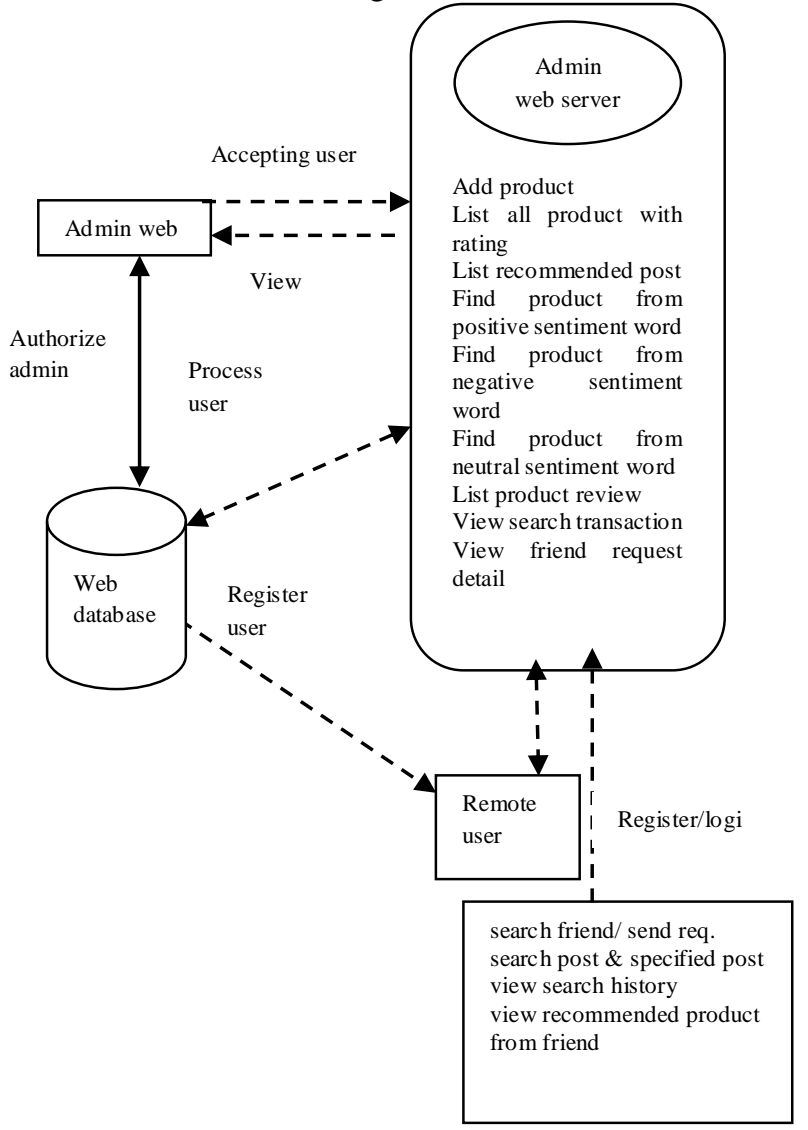

Fig.1. Framework for Customer Review Analysis

1. In the admin module, the Admin has to login by using valid user name and password.

2. After login successful he can do some operations such as add categories, add posts, list of all posts, list of all recommended posts, view good reviews, view bad reviews, list of all reviewed posts, list of users, list of all search history, update posts, by date wise, list of good reviews by date wise.

3. The admin can add the post by including product name, price, description and corresponding product image.

4. In the view all posts module, the admin can view the post by searching keyword and can get all the information about the product like product name, price, description and corresponding product image.

5. In the sentiment analytics module, the admin can analyze the sentiment based on products from positive sentiment words, products from negative sentiment words, products from neutral sentiment words and View Products Rating based on sentiment words.

\section{B. A Deep Learning Classification Approach for Short Messages Sentiment Analysis}

Peoples can communicate with each other through social media applications like whatsapp, facebook, twitter etc. social media apps get social media data from the applications and check what sentences are positive and negative sentiment using sentiment analysis. Deep learning methods like deep neural networks for using the Hindi tweets dataset and classifying them positive or negative sentiment polarity from twitter accounts.

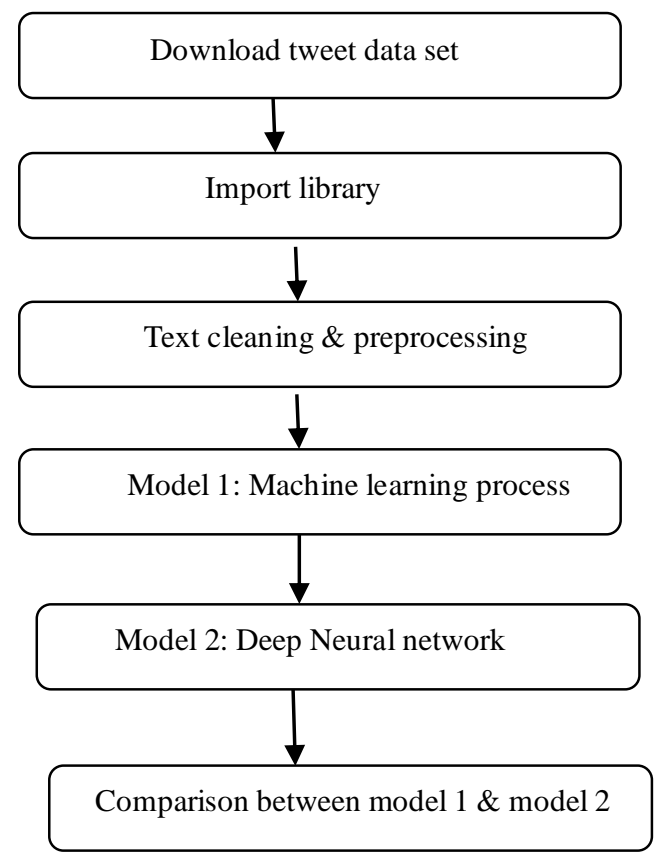

Fig.2. Social Media Sentiment Analysis Models

1. The Hindi dataset contains various posts in twitter.

2. The dataset is being used for text cleaning to get rid of the following things as follow[2][10]s:

- Those words which have same linguistic are combined together. This is stemming or lemmatization.

- All the text has been converted into lower case so that "the" and "The" will be consider as same word.

- Check all digits, stop word like etc, special symbol like \& has been removing it.

- In preprocessing of text cleaning: non characters from each sentence has been Cleaned,

- After this the sentences has been tokenized.

3. Using Machine Learning Algorithms: After cleaning the data, the following algorithm has been used.

- Algorithm for Logistic Regression

- Algorithm relates to Support Vector Machine (SVM).

- Random Forest

- XG Boost

4. After pre-modeling Neural Network recurrent technique which is also known as LSTM (Long Short Term Memory )[8][9] is used. This is help to remember the sequence of past data. Decision can be taken on the sentiment of the word. Keras is used for create a network. It has been built on tensor flow and it is helpful in most types of deep learning models. For estimating parameters such as dropout, no of cells have performed grid search with various parameter values. 
5. We found that deep neural network model[8] stood out with a greater accuracy and the machine learning model scans the negative sentences and gives a score of 0.34 while the machine learning algorithms give a score of $0.54[2]$.

C. Sentiment Analysis and Contrastive Experiments of Long News Texts

There are three methods are tested separately and the experimental results will be compared [3].

1. Dictionary-based Method: It is based on grammatical rules. Dictionary-based approaches are as follows:

2. Read the text data and segment the text.

3. Find the emotional words in the sentences and record the emotional values and positions.

4. Find the degree words before the emotional words. Set weights for degree words and multiply them by emotional values.

5. Look up the negative words before the emotional words and find out all the negative words. If then number is odd, the emotional value is multiplied by - 1 , and if it is even, the emotional value is multiplied by 1 .

6. Compute the emotional values of all clauses and record them in an array.

7. Calculate the positive emotional mean, negative emotional mean, positive emotional variance and negative emotional variance of each text.

8. Determine whether the comment is positive or negative by calculating the emotional score.

9. Based on Machine Learning: The machine learning method uses logistic regression, support vector machine (SVM) and other methods to classify the text. The final classification effect depends on the choice of training text and the correct emotional label. The main steps of the machine learning method are as follows:

Minimizing semantic granularity and using the two segment text.

Feature extraction, including feature selection and feature extraction. The steps of feature selection are as follows:

Calculate the information quantity of each word in the whole corpus by adding positive chi-square statistics to negative chi-square statistics of a word.
According to the amount of information, author rank the words in reverse order and select them with the highest information quantity as the characteristics.

10. Based on Deep Learning: Deep learning is suitable for word processing and semantic understanding because of the flexibility of deep learning structure. The underlying word embedding technology can avoid the processing difficulties caused by uneven text length. Using deep learning abstract features, you can avoid the work of a lot of manual extraction of features. Deep learning has local feature abstraction and memory functions that can simulate the connection between words and words [3].

D. Sentiment Analysis Using SVM and Deep Neural Network

The use of Support vector machines, embedding deep neural networks which are suitable for the high dimensional data analysis. This will act as the generalized automated review system for various industries Such as E- Commerce platform, YouTube comments, movie review rating etc. with more high precision and has potential to replace orthodox star-based rating System.

Support Vector Machine: Support vector machines (SVMs) are more of the rugged method for both classification and regression work, and have very good generalization performance. In DNN SVM

joined model for the estimation analysis. The CBOW show is utilized here to take in word installing from a huge gathering from the crude content. After that DNN demonstrate is acquainted with build disseminated sentence portrayals for the more extensive info information. At last, the appropriated sentence include portrayals are being utilized as the highlights for SVM[9] classifier preparing them by learning the likelihood circulation over marks [4].

\section{E. Sentiment Analysis of Movie Reviews Using Heterogeneous Features}

System identified sentiment orientation from review text documents using a hybrid approach. The hybrid approach means a combination of Machine learning and Lexicon-based (knowledge-based) approach [5].

Table I Gives the Comparative Study of deep learning for sementic analysis

TABLE I: COMPARATIVE STUDY OF DEEP LEARNING FOR SEMENTIC ANALYSIS

\begin{tabular}{|c|c|c|}
\hline Author & Description & Results \\
\hline $\begin{array}{l}\text { B.Seetharamulu et al. } \\
(2020)\end{array}$ & $\begin{array}{l}\text { Deep Learning for Sentiment Analysis Based of } \\
\text { Customer Reviews }\end{array}$ & $\begin{array}{l}\text { Better performance over existing } \\
\text { system. }\end{array}$ \\
\hline $\begin{array}{l}\text { Amit Kumar Goel et al } \\
(2020)\end{array}$ & $\begin{array}{l}\text { A Deep Learning Classification Approach for } \\
\text { Short Messages Sentiment Analysis }\end{array}$ & $\begin{array}{l}\text { The LSTM model scans the } \\
\text { negative sentences gives a score of } \\
0.34 \text { and the machine learning } \\
\text { algorithms give a score of } 0.54\end{array}$ \\
\hline
\end{tabular}




\begin{tabular}{ll}
\hline $\begin{array}{l}\text { Weinong Niu et al. } \\
\text { (2019) }\end{array}$ & $\begin{array}{l}\text { Sentiment Analysis and Contrastive } \\
\text { Experiments of Long News Texts }\end{array}$ \\
Punit Dubey et al (2019) & $\begin{array}{l}\text { Sentiment Analysis Using SVM and Deep } \\
\text { Neural Network }\end{array}$
\end{tabular}

Namita Mittal et al (2018)

Kruttika Jain et al(2018)

Rachana, Bandana et al. (2018)
Image Sentiment Analysis using Deep Learning

A Comparative Study of Machine Learning and Deep Learning Techniques for Sentiment Analysis

Sentiment Analysis of Movie Reviews Using Heterogeneous Feature

\author{
Average of the positive and negative \\ sample evaluations after ten-fold \\ cross-validation. \\ Sparse vector is \\ for solving movie reviews analysis \\ obtained by \\ Processing the review dataset of the \\ movies.
}

DNN, CNN, R-CNN, and Fast R$\mathrm{CNN}$ get optimum result

Reduce complexity

Heterogeneous features can get better results rather than using only machine learning or lexicon based features

\section{CONCLUSION}

In this paper, the study is presented on application of deep learning or machine learning for sentiment analysis. These techniques are applied in different application like twitter, Facebook, short message, images and movies data.

\section{REFERENCES}

[1] B. Seetharamulu, B. Naresh Kumar Reddy land K. Bramha Naidu, "Deep Learning for Sentiment Analysis Based of Customer Reviews," in IEEE, 2020.

[2] Amit Kumar Goel, Kalpana Batra, " A Deep Learning Classification Approach for Short Messages Sentiment Analysis," in IEEE 2020.

[3] Weinong Niu ,Lin Wu, " Sentiment Analysis and Contrastive Experiments of Long News Texts," IEEE 4th Advanced Information Technology, Electronic and Automation Control Conference (2019)

[4] Punit Dubey, Anshul Mishra, Bijoy Krishna Saha, "Sentiment Analysis Using SVM and Deep Neural Network, IEEE (2019).

[5] Namita Mittal, Divya Sharma, Manju Lata Joshi”, Image Sentiment Analysis using Deep Learning IEEE/WIC/ACM International Conference on Web Intelligence, 2018.

[6] Kruttika Jain, Shivani Kaushal " A Comparative Study of Machine Learning and Deep Learning Techniques for Sentiment Analysis," IEEE. 2018.

[7] Rachana Bandana, Nadiad "Sentiment Analysis of Movie Reviews Using Heterogeneous Feature,"IEEE 2019.

[8] Basri Ciftci, Mehmet Serkan Apaydin, "A Deep Learning Approach to Sentiment Analysisin Turkish," in IEEE,2018.

[9] Benwang Sun1, Fang Tian2, Li Liang1, "Tibetan Micro-Blog Sentiment Analysis Based on Mixed Deep Learning," IEEE,2018.

[10] Mohammed H. Abd El-Jawad, Rania Hodhod, and Yasser M. K. Omar, "Sentiment Analysis of Social Media Networks Using Machine Learning,IEEE,. 2018. 